\title{
SURFACES OF CONSTANT CURVATURE AND THEIR TRANSFORMATIONS*
}

\author{
BY
}

\section{LUTHER PFAHLER EISENHART}

BIANCHI $\uparrow$ has called attention to the fact that upon a surface of positive total curvature there is an infinity of conjugate systems of lines which are characterized by the property that when such a system is parametric the parameters can be chosen so that the second quadratic form becomes

$$
\lambda\left(d u^{2}+d v^{2}\right)
$$

where $\lambda$ is generally a function of both $u$ and $v$; BIANCHI calls such a system isothermal-conjugate. In a similar manner it can be shown $\ddagger$ that an infinity of conjugate systems exist on a surface of negative curvature such that the second quadratic form is reducible to

$$
\lambda\left(d u^{2}-d v^{2}\right)
$$

we call these also isothermal-conjugate. When the total curvature is constant and positive, the surface is called spherical. We find that when a spherical surface is referred to an isothermal-conjugate system the fundamental quantities for the surface and the equations which they satisfy take very simple forms forms which suggest transformations carrying the given surface into new spherical surfaces. This paper is given over to a study of these transformations.

In $\S 1$ we derive the fundamental equations for a spherical surface referred to isothermal-conjugate lines, show that its lines of curvature and characteristic lines $\S$ are isothermal-conjugate, derive the expressions for the spherical representation of the given isothermal system and for the fundamental functions when this given system is composed of the lines of curvature or the characteristic lines.

* Presented to the Society A pril 29, 1905. Received for publication January 10, 1905, and May 4, 1905.

† Lezioni, vol. I, p. 167 ; German translation by LUKaT, p. 135.

† Isothermal-conjugate systems of lines on surfaces, A merica n J o u r nal, vol. 25 (1903), p. 220.

\$ Three particular systems of ines on a surface, Transactions, vol. 5 (1904), p. 429. 
In $§ 2$ we discover a transformation which changes a spherical surface into a surface of the same curvature with the same second quadratic form as the given surface and the lines of curvature are in correspondence on the two surfaces. When the given surface is referred to its lines of curvature it is found that this is the transformation of Hazzidakis. * The new transformation is the transformation of HAzzidakis in a general form, for it can be shown that the latter is the only transformation of spherical surfaces leaving the total curvature and second quadratic forms unaltered and preserving lines of curvature. $\dagger$

An interesting example of the effect of the Hazzidakis transformation is afforded by applying it to the spherical surfaces of revolution. In addition to this study $\S 2$ is given over to the consideration of surfaces of constant mean curvature, which are always associated with spherical surfaces, as BONNET has shown.

In $\S 4$ another transformation is found which changes a spherical surface into another, leaving the total curvature and the second quadratic form unaltered, and changing lines of curvature into characteristic lines and vice versa. After a study of the effects of combinations of these and HAzzIDAKIs transformations, it is shown that these transformations or transformations of the two kinds are the only ones leaving the total curvature and second quadratic forms altered and interchanging the lines of curvature and characteristic lines.

In $\$ 5$ the pseudospherical surfaces are considered and their equations of condition are given forms analogous to those in $\$ 1$ for spherical surfaces, but one cannot find transformations similar to the above.

\section{\$1. Spherical surfaces referred to isothermal-conjugate lines.}

Consider a surface, $S$, of constant positive total curvature, $1 / a^{2}$. Let the parametric curves form an isothermal-conjugate system $\ddagger$ upon the surface and choose the parameters of these lines in such a way that

$$
D=D^{\prime \prime}, \quad D^{\prime}=0,
$$

where these $D$ 's are the coefficients of the second quadratic form of the surface. The Gauss $§$ and CodazzI \| equations can then be written in the respective forms

$$
D^{2}=H\left[\begin{array}{c}
\partial \\
\partial v
\end{array}\left(\begin{array}{c}
H \\
E
\end{array}\left\{\begin{array}{c}
11 \\
2
\end{array}\right\}\right)-\partial \ddot{\partial u}\left(\begin{array}{c}
H \\
E
\end{array}\left\{\begin{array}{c}
12 \\
2
\end{array}\right\}\right)\right]
$$

* Branchi, Lezioni, II, p. 436 ; German translation. p. 472.

† In a subsequent paper we shall show the part which the Hazzidakis transformation plays in the theory of the deformation of surfaces.

$\ddagger$ Bianch I, Lezioni, I, p. 167 ; German translation, p. 135.

\& Ibid., I, p. 93 ; p. 68.

|l Ibid., I, p. 119 ; p. 91. 
and

$$
\frac{\partial \log D}{\partial v}=\left\{\begin{array}{c}
12 \\
1
\end{array}\right\}-\left\{\begin{array}{c}
11 \\
2
\end{array}\right\}, \quad \frac{\partial \log D}{\partial v}=\left\{\begin{array}{c}
12 \\
2
\end{array}\right\}-\left\{\begin{array}{c}
22 \\
1
\end{array}\right\},
$$

where the Christofrel symbols $\left\{\begin{array}{c}r_{s} \\ t\end{array}\right\}$ are formed with respect to the linear element of $S$ written

$$
d s^{2}=E d u^{2}+2 F d u d v+G d v^{2},
$$

and we have put for the sake of brevity

$$
H=\sqrt{E^{\prime} G-F^{2}} .
$$

Since now

$$
K=\frac{L^{2}}{H^{2}}=\frac{1}{a^{2}},
$$

we have

$$
\begin{aligned}
& \frac{\partial \log D}{\partial v}=\frac{\partial \log H}{\partial v}=\left\{\begin{array}{c}
12 \\
1
\end{array}\right\}+\left\{\begin{array}{c}
22 \\
2
\end{array}\right\}, \\
& \frac{\partial \log D}{\partial u}=\frac{\partial \log H}{\partial u}=\left\{\begin{array}{c}
12 \\
2
\end{array}\right\}+\left\{\begin{array}{c}
11 \\
1
\end{array}\right\} .
\end{aligned}
$$

When these equations are combined with (3), the latter may be replaced by

$$
2 \frac{\partial F}{\partial u}=\frac{\partial E}{\partial v}-\frac{\partial G}{\partial v}, \quad 2 \frac{\partial F}{\partial v}=\frac{\partial G}{\partial u}-\frac{\partial E}{\partial u} .
$$

From this it follows that the functions $F$ and $E-G$ are solutions of the equation

$$
\frac{\partial^{2} \theta}{\partial u^{2}}+\frac{\partial^{2} \theta}{\partial v^{2}}=0
$$

The general integral of this equation is

$$
\theta=\Phi(u+i v)+\Psi(u-i v),
$$

where the functions $\Phi$ and $\Psi$ are arbitrary in form; moreover, in order that the surface be real $\Psi$ must be conjugate to $\Phi$. Consider this case and put

$$
F=\frac{\Phi(u+i v)+\Phi_{0}(u-i v)}{2}
$$

when $\Phi_{0}$ denotes the conjugate function of $\Phi$; from (7) it follows that

$$
E-G=i\left(\Phi-\Phi_{0}\right) \text {. }
$$

Since equations ( 7 ) have replaced the CodazzI equations, the only other equation of condition to be satisfied is the Gauss equation (2). Hence $\Phi$ can be chosen 
arbitrarily and there remains a partial differential equation of the second order to be satisfied by $G$; this equation is found by substituting the values for $D^{2}$, $F$, and $E$ in (2).

As equation ( 8$)$ is satisfied by $\theta=0$, it follows that a solution is

$$
F=0, \quad E-G=\text { const. }
$$

Since the latter constant is zero only in case $S$ is a sphere, we have the theorem :

The lines of curvature upon a spherical surface form an isothermal-conjugate system; these lines are isothermal-orthogonal only in case of the sphere.

Another solution of equations (7), suggested by the solution $\theta=0$ of equation $(8)$, is

$$
E=G, \quad F=\text { const. }
$$

where the constant is equal to zero only for the sphere. Now

$$
\frac{D}{E}=\frac{D^{\prime \prime}}{G} \quad D^{\prime}=0 .
$$

But the latter are the conditions that the conjugate parametric system is composed of the lines cutting one another under the minimum angle between conjugate lines;* Reina $\nmid$ has called the latter the characteristic lines. Hence the theorem :

The characteristic lines upon a spherical surface are isothermal-conjugate.

From the general expressions for the coefficients of the linear element of the spherical representation of a surface referred to a conjugate system, namely, $\$$

$$
\mathcal{E}=\frac{G D^{2}}{E G-H^{2}}, \quad \mathcal{F}=-\frac{F D D^{\prime \prime}}{E G-F^{2}}, \quad \mathcal{S}=\frac{E D^{\prime 2}}{E G-F^{2}},
$$

it is seen that for the present case

$$
\mathcal{E}=\frac{G}{a^{2}}, \quad \mathcal{F}=-\frac{F}{a^{2}}, \quad \mathcal{G}=\frac{E}{a^{2}} .
$$

In consequence of $(7)$ one has

$$
2 \frac{\partial \mathcal{F}}{\partial u}=\frac{\partial \mathcal{S}}{\partial v}-\frac{\partial \mathcal{G}}{\partial v}, \quad 2 \frac{\partial \mathcal{F}}{\partial v}=\frac{\partial \mathcal{G}}{\partial u}-\frac{\partial \mathcal{E}}{\partial u},
$$

that is, $\mathcal{S}, \mathcal{F}, \mathcal{G}$ satisfy equations $(7)$. Conversely, when $\mathcal{S}, \mathcal{F}, \mathcal{G}$ are three functions satisfying (12), one has

* Three particular systems of lines on a surface, Transactions, vol. 5 (1904), pp. 9, 443.

† Di alcune proprietd delle linee caratterische, Roma Accademia Lincei Rendiconti (4) V (1899), pp. 881-5.

$\ddagger$ BianchI, Lezioni, vol. I, p. 150 ; German translation, p. 120. 


$$
\left\{\begin{array}{c}
11 \\
1
\end{array}\right\}^{\prime}+\left\{\begin{array}{c}
22 \\
1
\end{array}\right\}^{\prime}=0, \quad\left\{\begin{array}{c}
22 \\
2
\end{array}\right\}^{\prime}+\left\{\begin{array}{c}
11 \\
2
\end{array}\right\}^{\prime}=0
$$

where the Christoffel symbols $\left\{\begin{array}{c}r s \\ t\end{array}\right\}^{\prime}$ are formed with respect to

$$
d \sigma^{2}=\varepsilon d u^{2}+2 \pi d u d v+\xi d v^{2} \text {. }
$$

Hence if $\mathcal{E}, \mathcal{F}, \mathcal{G}$ satisfy also the GaUss equation for the unit sphere, the parametric lines upon the sphere represent an isothermal-conjugate system of lines on a unique spherical surface. *

\section{§ 2. The transformation of Haxxidalis.}

The results of the last section suggest a transformation of spherical surfaces. Let $S$ be such a surface with the linear element (4), whose coefficients satisfy equations (7). We remark that the latter equations are satisfied also by $E_{1}$. $F_{1}, G_{1}$, where

$$
E_{1}=G, \quad F_{1}=-F, \quad G_{1}=E \text {. }
$$

If these values be subtituted in an equation similar to (2), namely,

$$
\left.D_{1}^{2}=H_{1}\left[\frac{\partial}{\partial v}\left(\begin{array}{c}
H_{1} \\
E_{1} \\
2
\end{array}\right\}\right)-\hat{c}\left(\begin{array}{c}
11 \\
\hat{c} u
\end{array}\left(\begin{array}{c|c}
I_{1} & 12 \\
E_{1} & 2
\end{array}\right\}\right)\right],
$$

when the Christoffel symbols are formed with respect to

$$
d s_{1}^{2}=E_{1} d u^{2}+2 F_{1} d u d u+G_{1} d v^{2}
$$

and one remarks that

$$
H_{1}=H
$$

it follows in consequence of (7) that this becomes

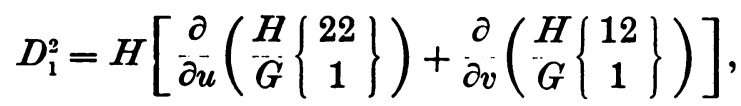

where now the symbols are formed with respect to (4). But the right-hand member of this equation is equivalent to the similar member of (2). $\dagger$ Hence, the GaUss equation is satisfied by taking $D_{1}=D_{1}^{\prime \prime}=D$. This gives the theorem :

When a spherical surface is referred to an isothermal-conjugate system and the parameters are chosen so that (1) is satisfied, there exists another spherical surface of the same total curvature and with the same second quadratic form as the given surfaces; the linear element is

$$
d s_{1}^{2}=G d u^{2}-2 F d u d v+E d v^{2}
$$

\footnotetext{
* Ibid., p. 168 ; p. 136.

† Ibid., p. 77 ; p. 53.
} 
It will be convenient to refer to the new surface $S_{1}$ as the conjugate of the given one. One remarks that the relation between the two surfaces is entirely reciprocal.

If the linear element of the spherical representation of $S_{1}$ is written

$$
d \sigma_{1}^{2}=\mathcal{E}_{1} d u^{2}+2{F_{1}}_{1} d u d v+\mathcal{S}_{1} d v_{1}^{2}
$$

it follows from (10), (11) and (13) that

$$
\mathcal{E}_{1}=\frac{E}{a^{2}}=\mathfrak{S}, \quad \mathscr{F}_{1}=-\mathcal{F}, \quad \mathcal{G}_{1}=\mathfrak{s} .
$$

Hence the coefficients of the spherical representation of $S$ and $S_{1}$ bear the same relation to one another as the first fundamental quantities of these surfaces.

A comparison of the formulas (11), (13) and (15) leads to the theorem :

$A$ spherical surface of unit total curvature is applicable to the unit sphere in such a way that an isothermal-conjugate system of lines on the former cor. respond to the spherical representation of the corresponding isothermal-conjugate system on its conjugate.

From the general equation of the lines of curvature

$$
\left(E D^{\prime}-F^{\prime} D\right) d u^{2}+\left(E D^{\prime \prime}-G D\right) d u d v+\left(F D^{\prime \prime}-G D^{\prime}\right) d v^{2}=0,
$$

it is seen that for both $S$ and $S_{1}$ the lines of curvature are given by

$$
F d u^{2}+(G-E) d u d v-F d v^{2}=0 .
$$

Hence the lines of curvature correspond upon a spherical surface and its conjugate.

Suppose that the isothermal-conjugate lines on $S$ are the lines of curvature: from (9) it is seen that we can put

$$
E=a^{2} \cosh ^{2} \theta, \quad G=a^{2} \sinh ^{2} \theta, \quad F=0
$$

or

$$
E=a^{2} \sinh ^{2} \theta, \quad G=a^{2} \cosh ^{2} \theta, \quad F=0
$$

according as the constant in (9) is positive or negative, $a^{2}$ being the absolute value of the constant.* Now in either case

and equation (2) becomes

$$
D=D^{\prime \prime}=a \sinh \theta \cosh \theta,
$$

$$
\frac{\partial^{2} \theta}{\partial u^{2}}+\frac{\partial^{2} \theta}{\partial v^{2}}+\sinh \theta \cosh \theta=0
$$

* Lezioni, vol. II., p. 436 ; p. 472. 
One sees that the values (18) lead to the conjugate of the surface whose first fundamental coefficients are given by (17) and vice versa. But this is the transformation which BIANCHI has called the transformation of HAzziddakis.

In order to show that the transformation which we have been considering in this section is the transformation of HAzzidAKIs in a more general form, we have only to prove the theorem:

The transformation of HAZZIDAKIs is the only one changing a spherical surface into one of the same curvature in such a way that the lines of curvature on the two surfaces correspond and the second quadratic forms are the same.

Let $S$ and $S_{1}$ be two surfaces of curvature $1 / a^{2}$ referred to their corresponding lines of curvature and let the second quadratic forms of the two surfaces be

$$
D\left(d u^{2}+d v^{2}\right) \text {. }
$$

If no distinction is made. with regard to homothetic surfaces, the most general integrals of equations (7) for $S$ and $S_{1}$ are

$$
E-G= \pm a^{2}, \quad E_{1}-G_{1}= \pm a^{2} .
$$

Since the total curvatures of $S$ and $S_{1}$ are equal and (19) is the second quadratic form of both surfaces, we must have also

$$
E_{1} G_{1}=E G
$$

In conformity with this we put

$$
E_{1}=\lambda E, \quad G_{1}=\frac{1}{\lambda} G
$$

Substituting these values in the second of equations (20) and taking account of the first, we get

$$
\lambda^{2} E \mp a^{2} \lambda+\left( \pm a^{2}-E\right)=0,
$$

of which the solutions are

$$
\lambda= \pm 1, \quad \pm \frac{G}{E}
$$

Since $E_{1}$ and $G_{1}$ must be positive, the only possibility is

$$
E_{1}=G, \quad G_{1}=E,
$$

which proves the theorem. Hence the Hazzidakis transform, or conjugate, of a spherical surface may be defined to be the surface with the same total curvature and second quadratic form and such that the lines of curvature on the two surfaces are in correspondence.

We shall find that on the two surfaces $S$ and $S_{1}$ the characteristic lines also 
are in correspondence. For, the general equation of the characteristic lines upon a surface referred to a conjugate system is *

$$
D\left(G D-E D^{\prime \prime}\right) d u^{2}-4 F D D^{\prime \prime} d u d v+D^{\prime \prime}\left(E D^{\prime \prime}-G D\right) d v^{2}=0 \text {. }
$$

When the system is such that the second quadratic form is (19), this reduces to

$$
(G-E) d u^{2}-4 F d u d v+(E-G) d v^{2}=0,
$$

which in consequence of (13) is the equation of these lines on $S_{1}$ also. Hence the characteristic lines correspond upon two conjugate spherical surfaces.

\section{§3. Surfaces of revolution and surfaces of constant mean curvature.}

The spherical surfaces of revolution, referred to their meridians and parallels, lend themselves readily to the transformation of Hazzidakis. Bianchi $\dagger$ shows that these surfaces are defined by the equations

$$
x=r \cos w, \quad y=r \sin w, \quad z=\phi(r),
$$

where

$$
r=\kappa \cos u, \quad z=\int \sqrt{1-\kappa^{2} \sin ^{2} u} d u,
$$

in which $\kappa$ denotes a constant. When $\kappa$ is equal to one, $S$ is a sphere of unit radius. The other surfaces of this kind are divided into two classes according as $\kappa$ is less or greater than one. From (23) and (24) it is found that the linear element of $S$ has the form

$$
d s^{2}=\frac{d r^{2}}{\kappa^{2}-r^{2}}+r^{2} d w^{2}
$$

and for the second quadratic form we have

$$
\Phi=r \sqrt{1-\kappa^{2}+r^{2}}\left[\frac{d r^{2}}{\left(\kappa^{2}-r^{2}\right)\left(1-\kappa^{2}+r^{2}\right)}+d w^{2}\right] .
$$

As the case of the sphere does not afford any particular interest, we pass to the consideration of the two remaining cases in turn.

$1^{\circ}$. $\kappa<1$. In order to have $D=D^{\prime \prime}$ we change the parameters, putting

$$
d t=\frac{d r}{\sqrt{\left(1-\kappa^{2}+r^{2}\right)\left(\kappa^{2}-r^{2}\right)}} .
$$

Upon integration we have $r=\kappa c n t$, with $\kappa$ the modulus. When this change is effected upon (25) and (26), they become

* Three particular systems of lines, 1. c., p. 429.

† L. c., vol. 1, p. 233 ; German translation, p. 197. 


$$
\begin{aligned}
& d s^{2}=d n^{2} t d t^{2}+\kappa^{2} c n^{2} t d w^{2} \\
& \Phi=\kappa \operatorname{cnt} d n t\left(d t^{2}+d u^{2}\right) .
\end{aligned}
$$

$2^{\circ} . \kappa_{1}>1$. For this case we put

$$
\frac{d t}{\kappa_{1}}=\frac{d r}{\sqrt{\left(1-\kappa_{1}^{2}+r^{2}\right)\left(\kappa^{2}-r^{2}\right)},}
$$

which gives by integration $r^{2}=\kappa_{1}^{2}-s n^{2} t$, and the modulus is $1 / \kappa_{1}$. Now the linear element and second quadratic form, after putting $w_{1}=\kappa_{1} w$, reduce to

$$
\begin{aligned}
& d s^{2}=\frac{c n^{2} t}{\kappa_{1}^{2}} d t^{2}+d n^{2} t d w_{1}^{2}, \\
& \Phi=\stackrel{c n t \cdot d n t}{\kappa_{1}}\left(d t^{2}+d w_{1}^{2}\right) .
\end{aligned}
$$

If we take for $\kappa_{1}$ the inverse of $\kappa$, the elliptic functions have the same modulus. Again, if we take the same $t$ for both cases and to a line $w=$ const, on one of the first case make correspond a line $\omega_{1}=$ const, on a surface of the second kind, the forms (28) and (30) are the same, and (30) is obtained by interchanging the coefficients in (28) and vice versa. Hence:

Given a surface of revolution of constant positive curvature corresponding to a certain $\kappa$; its conjugate is the similar surface corresponding to $1 / \kappa$.

BoNNET has shown that there is a surface of constant mean curvature $1 / 2 a$ parallel to a surface of total curvature $1 / u^{2}$ and at the distance $a$. If the parametric curves be the lines of curvature for these surfaces and the fundamental functions for the former surface be denoted by $e, g, d, d^{\prime \prime}$, the following relations hold : *

where

$$
\begin{gathered}
e=y=\lambda a\left[a\left(\begin{array}{l}
1 \\
\rho_{1}
\end{array}+\frac{1}{\rho_{2}}\right)-2\right], \\
d=\frac{e}{a}-\lambda\left[a\left(\begin{array}{l}
1 \\
\rho_{1}
\end{array}+\frac{1}{\rho_{2}}\right)-1\right], \quad d^{\prime \prime}=\frac{g}{a}-\lambda\left[a\left(\frac{1}{\rho_{1}}+\frac{1}{\rho_{2}}\right)-1\right],
\end{gathered}
$$

$$
\lambda=d=d^{\prime \prime} \text {. }
$$

Let $S$ and $S_{1}$ be two surfaces of unit total curvature which are the transforms of one another and denote by $S$ and $S_{1}$ the two surfaces of constant mean curvature respectively which are associated with the former, as remarked by the theorem of BonNet. Now $a$ in the above formulæ has the value unity. If the functions for $S_{1}$ be denoted by $E_{1}, G_{1}, D_{1}, D_{1}^{\prime \prime}$, the above relations lead to the following in consequence of the relation between $S$ and $S_{1}$ :

$$
e=g=e_{1}=g_{1}, \quad d=d_{1}^{\prime \prime}, \quad d^{\prime \prime}=d_{1} .
$$

${ }^{*}$ KNoblaUCh, Einleitung in die allgemeine Theorie der krummen Flächen, p. 234. 
We have then the theorem :

Given any surface of constant mean curvature, there is another surface of this kind applicable to it in such a way that lines of curvature correspond; when the latter are parametric and the parameters are suitably chosen

$$
D=D_{1}^{\prime \prime}, \quad D^{\prime \prime}=D_{1} .
$$

BONNET * has shown that every surface of constant mean curvature is applicable to an infinity of surfaces of the same mean curvature with preservation of the principal radii. It has just been seen that to every surface of this kind there is applicable a surface of the same mean curvature with preservation of the lines of curvature and equality of the principal radii in inverse order. Hence the theorem :

Given a surface $S$ of constant mean curvature, there are two families of surfaces of the same mean curvature applicable to it; for the surfaces of the one family the principal radii are equal to the corresponding radii of $S$, and for the surfaces of the other fumily the principal radii are equal to those of $S$ in reverse order.

\section{§4. A new transformation of spherical surfaces.}

Let $E, F, G$ be three functions satisfying equations (7). These equations are satisfied also by

$$
E_{1}=\frac{E+G-2 F}{2}, F_{1}=\frac{E-G}{2}, G_{1}=\frac{E+G+2 F^{\gamma}}{2} .
$$

We find that

$$
E_{1} G_{1}-F_{1}^{2}=E G-F^{2}
$$

so that we are led to inquire whether there exists a surface $S_{1}$ whose first fundamental coefficients are given by (31) and whose second quadratic form is the same as for $S$. In order that this be true, it is necessary and sufficient that these functions satisfy the GaUss equation $\left(13^{\prime}\right)$, which in consequence of $(6)$, (31) and (32) can be given the form

$$
\begin{aligned}
4 H a^{2} & =\left\{\frac{\partial}{\partial v} \log (E+G-2 F) \frac{\partial}{\partial u}\left(\frac{E-G}{H}\right)-\frac{\partial}{\partial u}\left[\frac{1}{H} \frac{\partial}{\partial u}(E+G+2 F)\right]\right. \\
& \left.-\frac{\partial}{\partial v}\left[\frac{1}{H} \frac{\partial}{\partial v}(E+G+2 F)\right]-\frac{\partial}{\partial u} \log (E+G-2 F) \frac{\partial}{\partial v}\left(\frac{E-G}{H}\right)\right\} .
\end{aligned}
$$

* Mémoire sur la théorie des surfaces applicables sur une surface donnée, Jou rnal de J'École Polytechnique, Cahier 42, p. 77. 
The Gauss equation for $S$ can be reduced to either of the equivalent forms

$$
\begin{aligned}
& 2 a^{2} H=\frac{1}{E} \frac{\partial E}{\partial v} \frac{\partial}{\partial u}\left(\frac{F}{H}\right)-\frac{\partial}{\partial u}\left(\frac{1}{H} \frac{\partial G}{\partial u}\right)-\frac{\partial}{\partial v}\left(\frac{1}{H} \frac{\partial G}{\partial v}\right)-\frac{1}{E} \frac{\partial E}{\partial u} \frac{\partial}{\partial v}\left(\frac{F}{H}\right) \\
& 2 a^{2} H=\frac{1}{G} \frac{\partial G}{\partial u} \frac{\partial}{\partial v}\left(\frac{F}{H}\right)-\frac{\partial}{\partial v}\left(\frac{1}{H} \frac{\partial E}{\partial v}\right)-\frac{\partial}{\partial u}\left(\frac{1}{\bar{H}} \frac{\partial E}{\partial u}\right)-\frac{1}{G} \frac{\partial G}{\partial v} \frac{\partial}{\partial u}\left(\frac{F}{H}\right)
\end{aligned}
$$

If these equations be added and the result be subtracted from (33), we will be brought to an equation which vanishes identically in consequence of (7). Hence :

Given a spherical surface referred to an isothermal-conjugate system of lines and let the parameters be so chosen that the second fundamental functions $D$ and $D^{\prime \prime}$ are equal ; there exists a second spherical surface with the same second quadratic form and the coefficients of its linear element are given by (31). For convenience we shall call the new surface $S_{1}$ the adjoint of $S$. When the expressions (31) are substituted in the equation of the characteristic directions on $S_{1}$, namely (22)

$$
\left(G_{1}-E_{1}\right) d u^{2}-4 F_{1} d u d v+\left(E_{1}-G_{1}\right) d v^{2}=0,
$$

this equation reduces to (16). Hence the characteristic lines on $S_{1}$ correspond to the lines of curvature on $S$. And the equation of the lines of curvature on $S_{1}$ reduces to (22). Hence upon a spherical surface and its adjoint the lines of curvature of one surface correspond to the characteristic lines upon the other.

For the spherical representation of $S_{1}$ we have

$$
\mathcal{E}_{1}=\frac{G_{1}}{a^{2}}, \quad \mathcal{F}_{1}=\frac{-F_{1}}{a^{2}}, \quad \mathcal{S}_{1}=\frac{E_{1}}{a^{2}},
$$

which in consequence of (31) and (11) may be written

$$
\mathcal{E}_{1}=\frac{\mathcal{E}+\mathcal{G}-2 \mathcal{F}}{2}, \quad \mathcal{F}_{1}=\frac{\mathcal{E}-\mathcal{G}}{2}, \quad \mathcal{G}_{1}=\frac{\mathcal{E}+\mathcal{G}+2 \mathcal{F}}{2} .
$$

Hence the coefficients of the spherical representation of corresponding isothermal-conjugate lines on a spherical surface and its adjoint bear the same relation to one another as the first fundamental functions for these surfaces.

For the sake of convenience we shall refer to the transformation of HAzzIDAKIs and the one treated in the preceding section as the transformations $H$ and $I$ respectively. First of all we remark that throughout any number of operations of $H$ and $I$ separately or conjointly the second quadratic form is invariant. Since the relation between a surface and its transform by either $H$ or $I$ is completely reciprocal, it follows from (13) and (31) that the same sur- 
face is obtained when $H$ and $I$ are applied consecutively as when their order is reversed. And also that one is brought back to the original surface by any one of the sets of combined transformations

$$
H H, H I I, \quad I H I, \quad I I H \text {. }
$$

Denote by $T$ any transformation of a spherical surface into a second surface of this kind which preserves the total curvature and second quadratic form and interchanges lines of curvature and characteristic lines. Then $I T$ leaves lines of curvature unaltered and consequently is either identity or an $H$ transformation. Therefore $T$ is either an $I$ transformation or a combination of an $I$ and one or more $H$ transformations. Hence the theorem :

The only transformation which changes a spherical surface into a spherical surface of the same curvature and the same second quadratic form and interchanges the lines of curvature and characteristic lines is the I transformation or a combination of the latter and Hazzidakis transformations.

\section{§5. Surfaces of negative curvature.}

In this section we study surfaces of negative curvature in a manner similar to the preceding. We have shown * that upon such a surface there is an infinity of conjugate systems such that, when these lines are taken as parametric, the parameters can be so chosen that

$$
D=-D^{\prime \prime}, \quad D^{\prime}=0 .
$$

The Gauss equation can be written

$$
K=\frac{1}{H}\left[\frac{\partial}{\partial v}\left(\begin{array}{c}
H \\
E
\end{array}\left\{\begin{array}{c}
11 \\
2
\end{array}\right\}\right)-\frac{\partial}{\partial u}\left(\frac{H}{E}\left\{\begin{array}{c}
12 \\
2
\end{array}\right\}\right)\right],
$$

and proceeding as in $\S 1$ we find that if we put

$$
K=-\frac{D^{2}}{H^{2}}=-1,
$$

the Codazzi equations can be put in the form

$$
2 \frac{\partial F}{\partial u}=\frac{\partial E}{\partial v}+\frac{\partial G}{\partial v}, \quad 2 \frac{\partial F}{\partial v}=\frac{\partial E}{\partial u}+\frac{\partial G}{\partial u} .
$$

From this it is seen that $F$ and $E+G$ are solutions of

$$
\frac{\partial^{2} \theta}{\partial u^{2}}-\frac{\partial^{2} \theta}{\partial v^{2}}=0
$$
220.

* Isothermal-conjugate Systems, A merioan Journal of Matbematios, vol. 25 (1903), p. 
When either of these functions is known, the other is given by a quadrature from (38) and then it remains to find $E$ or $G$ from (36). This shows that every solution of (39) leads to surfaces of constant negative curvature. The most general form which $F$ can have is

$$
F=\frac{\Phi(u+v)+\Psi(u-v)}{2},
$$

and then

$$
E+G=\Phi(u+v)-\Psi(u-v) .
$$

It is clear that equations (38) are satisfied by

$$
E_{1}=G, \quad G_{1}=E, \quad F_{1}=F .
$$

When these values of $E, G, F$ are substituted in (36) with $K$ replaced by -1 , and it is noted that they satisfy (38), we get

$$
\left[\frac{\partial}{\partial u}\left(\frac{H_{1}}{G_{1}}\left\{\begin{array}{c}
22 \\
1
\end{array}\right\}_{1}\right)-\frac{\partial}{\partial v}\left(\begin{array}{c}
H_{1} \\
G_{1}^{\prime}
\end{array}\left\{\begin{array}{c}
12 \\
1
\end{array}\right\}\right)\right]=1,
$$

where the Christoffel symbols $\left\{\begin{array}{c}r s \\ t\end{array}\right\}_{1}$ are formed with respect to

$$
E_{1} d u^{2}+2 F_{1} d u d v+G_{1} d v^{2}
$$

But the left-hand side of the above expression is equal to the total curvature of the surface with the linear element (41)* Hence when these coefficients have the values $(40)$, the surface $S_{1}$ has positive curvature, so that for pseudospherical surfaces a transformation analogous to the transformation of HAzzidakis does not exist. The linear element of the spherical representation of $S$ is readily found to be $\dagger$

$$
d \sigma^{2}=G d u^{2}+2 F d u d v+E d v^{2}
$$

which is the same as (41) when the coefficients in the latter have the values given by (40); this accounts for the above result.

It is of interest to determine whether there is a surface with total curvature equal to one whose linear element takes this form when the parametric lines form an isothermal conjugate system. The conjugate of this surface would have for linear element

$$
d s^{2}=E d u^{2}-2 F d u d v+G d v^{2},
$$

so that $E, F, G$ would have to satisfy equations

$$
2 \frac{\partial F}{\partial u}=\frac{\partial G}{\partial v}-\frac{\partial E}{\partial v}, \quad 2 \frac{\partial F}{\partial v}=\frac{\partial E}{\partial u}-\frac{\partial G}{\partial u},
$$

* Bia NCHI, l. c., vol. I., p. 77 ; German translation, p. 53.

† Ibid, p. 150 ; p. 120. 
as well as (38). For these to be satisfied it is necessary that $E$ be a function of $u$ alone and $G$ of $v$ alone. In consequence of (42), we have for the forms of these functions

$$
E=\alpha u^{2}+2 \gamma u+\epsilon, \quad F=\alpha u v+\beta u+\gamma v+\delta, \quad G=\alpha v^{2}+2 \beta v+\kappa,
$$

where $\alpha, \beta, \gamma, \delta, \epsilon$ and $\kappa$ are constants. For no values of these constants does the formula (36) make $K$ equal to -1 , which shows the non-existence of such a transformation.

One sees also that if $E, G, F$ are solutions of equations (38) so also are

$$
E_{1}=\frac{E-G+2 F}{2}, \quad G_{1}=-\frac{G-E+2 F}{2}, \quad F_{1}=\frac{E+G}{2} .
$$

But now

$$
E_{1} G_{1}-F_{1}^{2}=-\left(E G-F^{2}\right)
$$

hence the transform is imaginary-

Princeton University. 